\title{
O gênero Micrasterias Agardh ex Ralfs (Desmidiaceae, Zygnemaphyceae) em um lago artificial urbano, Paraná, Brasil
}

\author{
Carina Moresco ${ }^{1,3}$, Stefania Biolo $^{1}$ e Norma Catarina Bueno ${ }^{2}$
}

Recebido: 23.10.2008; aceito: 04.06.2009

\begin{abstract}
The genus Micrasterias Agardh ex Ralfs (Desmidiaceae, Zygnemaphyceae) in an urban artificial lake, Paraná State, Brazil). This study aimed to identify the species belonging to the genus Micrasterias from "Lago Municipal de Cascavel" and contributes to the knowledge of its taxonomic composition and geographic distribution in Paraná State. Taxonomic survey was based on monthly samples, performed from January 2002 to October 2003. Samples were collected using plankton net ( $25 \mu \mathrm{m}$ mesh size) and immediately fixed in Transeau solution. Qualitative analysis and illustrations were conducted with the aid of an optical microscope with drawing tube. Nine taxa were recorded: Micrasterias borgei Krieger var. borgei, Micrasterias denticulata Brébisson ex Ralfs var. denticulata, Micrasterias jenneri Ralfs var. jenneri, Micrasterias laticeps Nordstedt var. laticeps, Micrasterias laticeps Nordstedt var. acuminata Krieger, Micrasterias quadridentata (Nordstedt) Grönblad, Micrasterias rotata (Greville) Ralfs var. rotata, Micrasterias thomasiana Archer var. notata (Nordstedt) Grönblad and Micrasterias truncata (Corda) Brébisson ex Ralfs var. truncata. Micrasterias jenneri var. jenneri and Micrasterias quadridentata are cited by first time in Paraná State.
\end{abstract}

Key words: Cascavel Municipal Lake, desmids, phytoplankton, taxonomy

RESUMO - (O gênero Micrasterias Agardh ex Ralfs (Desmidiaceae,Zygnemaphyceae) em um lago artificial urbano, Paraná, Brasil). Este trabalho visou identificar as espécies do gênero Micrasterias no Lago Municipal de Cascavel e contribuir para a compreensão da sua taxonomia e distribuição geográfica no Estado do Paraná. O levantamento taxonômico foi baseado em amostras coletadas mensalmente de janeiro de 2002 a outubro de 2003. As amostras foram obtidas através de rede de plâncton (25 $\mu \mathrm{m}$ de malha) e imediatamente fixadas em solução de Transeau. A análise qualitativa e as ilustrações procederam-se sob microscópio óptico acoplado à câmara clara. Foram registrados nove táxons: Micrasterias borgei Krieger var. borgei, Micrasterias denticulata Brébisson ex Ralfs var. denticulata, Micrasterias jenneri Ralfs var. jenneri, Micrasterias laticeps Nordstedt var. laticeps, Micrasterias laticeps Nordstedt var. acuminata Krieger, Micrasterias quadridentata (Nordstedt) Grönblad, Micrasterias rotata (Greville) Ralfs var. rotata, Micrasterias thomasiana Archer var. notata (Nordstedt) Grönblad e Micrasterias truncata (Corda) Brébisson ex Ralfs var. truncata. Micrasterias jenneri var. jenneri e Micrasterias quadridentata são primeiras citações no Paraná.

Palavras-chave: desmídeas, fitoplâncton, Lago Municipal de Cascavel, taxonomia

\section{Introdução}

Apenas cinco trabalhos exclusivamente taxonômicos registraram a ocorrência do gênero Micrasterias Agardh ex Ralfs (classeZygnemaphyceae) para o Estado do Paraná. Bittencourt-Oliveira \& Mecenas (1994) inventariaram 14 táxons de Micrasterias no fitoplâncton do rio Tibagi. Cecy et al. (1997) registraram três espécies entre as desmídias do reservatório do rio Passaúna em Curitiba. No mesmo ambiente, Picelli-Vicentim et al. (2001) registraram somente Micrasterias truncata (Corda) Brébisson ex Ralfs var. pusilla G.S. West. Biolo et al. (2008) documentaram cinco táxons de Micrasterias no rio São Francisco Falso, um tributário do reservatório de Itaipu, e finalmente Felisberto \& Rodrigues (2008) encontraram sete espécies de Micrasterias no reservatório de Salto do Vau (Bacia do rio Iguaçu, Paraná). Os trabalhos de Andrade \& Rachou (1954), Lozovei \& Luz (1976), Bittencourt-Oliveira (2002), Felisberto \& Rodrigues (2005), Algarte et al. (2006), incluem táxons identificados apenas em nível genérico ou listagens de táxons em trabalhos de cunho ecológico.

1. Universidade Estadual de Maringá, Departamento de Biologia, Nupélia, Av. Colombo, 5790, 87020-900 Maringá, PR, Brasil

2. Universidade Estadual do Oeste do Paraná, Centro de Ciências Biológicas e da Saúde, Rua Universitária, 2069, 85814-110 Cascavel, PR, Brasil

3. Autor para correspondência: camoresco@hotmail.com 
O Lago Municipal de Cascavel é um reservatório artificial e juntamente com o Jardim Zoológico formam o Parque Paulo Gorski, importante manancial abastecedor e local de visitação turística. Até o momento apenas os trabalhos de Tavares \& Moreira (2000) e Moresco \& Bueno (2007) fornecem respectivamente, informações acerca das classes Bacillariophyceae e Chlorophyceae (Scenedesmus Meyen e Desmodesmus An, Friedl \& Hegewald).

O presente trabalho teve como objetivo o estudo taxonômico das espécies de Micrasterias no Lago Municipal de Cascavel, bem como contribuir com a distribuição das espécies no Estado do Paraná.

\section{Material e métodos}

O Lago Municipal de Cascavel (248's e $53^{\circ} 28^{\prime} \mathrm{W}$ ) está inserido na bacia hidrográfica do rio Cascavel (figura 1), abastecido por vários córregos, com área de drenagem de $117,5 \mathrm{~km}^{2}$. Localiza-se em clima subtropical úmido, mesotérmico e sem estação seca definida (Itcf 1990). Trata-se de um reservatório artificial destinado ao abastecimento público e constitui-se num importante local de visitação turística. Nos últimos dez anos, o lago vem sofrendo assoreamento de suas margens devido à ocupação habitacional de seu entorno, além de receber descargas de fontes clandestinas de esgoto.

As coletas do material foram realizadas mensalmente, no período de janeiro de 2002 a outubro de 2003, na região litorânea. Uma rede de plâncton com malha de $25 \mu \mathrm{m}$ foi utilizada para a coleta das amostras, as quais foram imediatamente fixadas em solução Transeau, na proporção 1:1 (Bicudo \& Menezes 2006). Para as análises qualitativas, foram preparadas lâminas temporárias, em média 10 lâminas por amostra ou até não ocorrerem táxons diferentes. A análise das lâminas e as ilustrações foram realizadas com microscópio óptico binocular e câmara clara, em aumentos de 400 e 1000 x. As amostras estão depositadas no Herbário da Universidade Estadual de Maringá (HUEM), sob a numeração 11966 a 11987. A identificação dos táxons foi baseada em bibliografia clássica e especializada.

\section{Resultados e Discussão}

O levantamento florístico do gênero Micrasterias, no Lago Municipal de Cascavel registrou a ocorrência de nove táxons: Micrasterias borgei Krieger var. borgei, Micrasterias denticulata Brébisson ex Ralfs var. denticulata, Micrasterias jenneri Ralfs var. jenneri, Micrasterias laticeps Nordstedt var. laticeps, Micrasterias laticeps Nordstedt var. acuminata Krieger, Micrasterias quadridentata (Nordstedt) Grönblad, Micrasterias rotata (Greville) Ralfs var. rotata, Micrasterias thomasiana Archer var. notata (Nordstedt) Grönblad e Micrasterias truncata (Corda) Brébisson ex Ralfs var. truncata. Micrasterias jenneri var. jenneri e Micrasterias quadridentata são primeiras citações para o Estado do Paraná.

\section{Micrasterias Agardh ex Ralfs 1848}

Células solitárias (filamentosas apenas em $M$. foliacea Bailey ex Ralfs) de contorno elíptico à circular, achatadas frontalmente e com constrição mediana profunda. Cada semicélula pode ser dividida em até cinco lobos, apresenta quatro profundas incisões que são simetricamente posicionadas no eixo da célula. O lobo apical é alongado ou lateralmente expandido, mas nunca subdividido, com lobos basais e laterais geralmente subdivididos em lóbulos através de incisões, possibilitando a formação de lóbulos de até quinta ordem. A parede celular é lisa, pontuada ou ornamentada com espinhos e protuberâncias. O cloroplasto é axial e os pirenóides são esparsos. As espécies pertencentes a este gênero possuem elevado polimorfismo e muitas são cosmopolitas.

Chave artificial para identificação das espécies e variedades do gênero Micrasterias

1. Semicélula 3-lobadas

2. Extremidades dos lobos basais acuminados M. laticeps var. acuminata

2. Extremidades dos lobos basais 2-denticulados M. laticeps var. laticeps

1. Semicélula 5-lobadas

3. Parede celular com espinhos M. borgei var. borgei

3. Parede celular lisa ou pontuada

4. Lobo apical curto e alargado 
5. Incisões entre os lobos apicais e laterais estreitas, lineares; lobos basais e laterais subdivididos em 2 lóbulos retos ou chanfrados M. jenneri var. jenneri

5. Incisões entre os lobos apicais e laterais abertas, acutangulares; lobos basais e laterais subdivididos em 2 lóbulos chanfrados, 2-denticulados M. truncata var. truncata

4. Lobo apical alongado e estreito

6. Lobo apical com ângulos aproximadamente arredondados M. denticulata var. denticulata

6. Lobo apical com ângulos denticulados

7. Contorno da célula circular; lobos basais e laterais subdivididos em lóbulos com ápices truncados M. quadridentata

7. Contorno da célula subcircular; lobos basais e laterais subdivididos em lóbulos com ápices 2-denticulados

8. Lobo basal com 4 lóbulos; margem superior do lobo apical levemente convexa a reta M. rotata var. rotata

8. Lobo basal com 8 lóbulos ou mais; margem superior do lobo apical com uma incisão arredondada M. thomasiana var. notata

Micrasterias borgei Krieger var. borgei, Rabenhorst Kryptogamen-Fl. Deutschl. 13(2): 86. 128. 1939.

Figuras 2-3

Células de contorno elíptico a elíptico-oblongo, 1,2-1,4 vezes mais longa que larga, 194,5-302,7 $\mu \mathrm{m}$ compr., 140,5-245,9 $\mu \mathrm{m}$ larg., istmo 24,3-72,9 $\mu \mathrm{m}$; semicélula 5-lobada, incisões interlobulares acutangulares, constrição mediana profunda; lobos basais e laterais separados por uma incisão linear, acutangular na porção distal; lobos basais subdivididos em 2-4 lóbulos por 1-3 incisões, acutangulares, levemente dilatados na extremidade, 2-4 denticulados; lobos laterais subdivididos em 4-8 lóbulos por 3-7 incisões, acutangulares, 2-4 denticulados; lobo apical alongado, estreito, estendendo-se levemente além dos lobos laterais, margens retas ou levemente convexas, abrindo-se para o ápice chanfrado, ângulos projetados formando processos divergentes 2-denticulados, curvados ou não, divergentes; parede celular ornamentada com espinhos, curtos.

Material examinado: BRASIL. Paraná: Cascavel, Lago Municipal de Cascavel, 26-I-2002, C. Moresco \& N.C.Bueno s.n.(HUEM11966); idem, 24-II-2002, C. Moresco \& N.C. Bueno s.n. (HUEM11967); idem, 24-V-2002, C. Moresco \& N.C. Bueno s.n. (HUEM11970); idem, 21-VI-2002, C. Moresco \& N.C. Bueno s.n. (HUEM11971); idem, 27-X-2002, C. Moresco \& N.C. Bueno s.n. (HUEM11975); idem, 30-XI-2002, C. Moresco \& N.C. Bueno s.n. (HUEM11976); idem, 15-XII-2002, C. Moresco \& N.C. Bueno s.n. (HUEM11977); idem, 26-I-2003, C. Moresco \& N.C. Bueno s.n. (HUEM11978); idem, 27-IV-2003, C. Moresco \& N.C. Bueno s.n.
(HUEM11981); idem, 25-V-2003, C. Moresco \& N.C. Bueno s.n. (HUEM11982); idem, 28-VI-2003, C. Moresco \& N.C. Bueno s.n. (HUEM11983); idem, 27-VII-2003, C. Moresco \& N.C. Bueno s.n. (HUEM11984); idem, 31-VIII-2003, C. Moresco \& N.C. Bueno s.n. (HUEM11985).

Ocorrência no Paraná: Rio Tibagi (BittencourtOliveira \& Mecenas 1994); Rio Tibagi, Municípios de Ipiranga e Telêmaco Borba (Bittencourt-Oliveira 2002); Reservatório de Rosana, Município de Diamante do Norte (Felisberto \& Rodrigues 2005).

Bittencourt-Oliveira \& Mecenas (1994) encontraram variação morfológica na projeção do lobo apical dos indivíduos registrados. Tal variação não foi registrada nos indivíduos do presente trabalho.

Micrasterias denticulata Brébisson ex Ralfs var. denticulata, Brit. Desm. 70. 1848.

Figura 4

Célula de contorno elíptico-oblongo, 1,1-1,4 vezes mais longa que larga, 143,2-264,8 $\mu \mathrm{m}$ compr.,

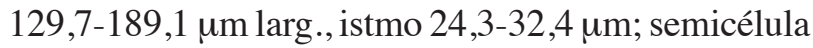
5-lobada, incisões interlobulares lineares, estreitas, constrição mediana profunda; lobos basais e laterais cuneados, separados por uma incisão linear; lobos basais subdivididos em 2 lóbulos chanfrados; lobos laterais subdivididos em 4 lóbulos por 3 incisões, chanfrados; lobo apical estreito, alongado, estendendo-se levemente além dos lobos laterais, margens retas ou levemente côncavas, abrindo-se para o ápice chanfrado profundamente, ângulos arredondados, não projetados; parede celular finamente ou grosseiramente pontuada. 


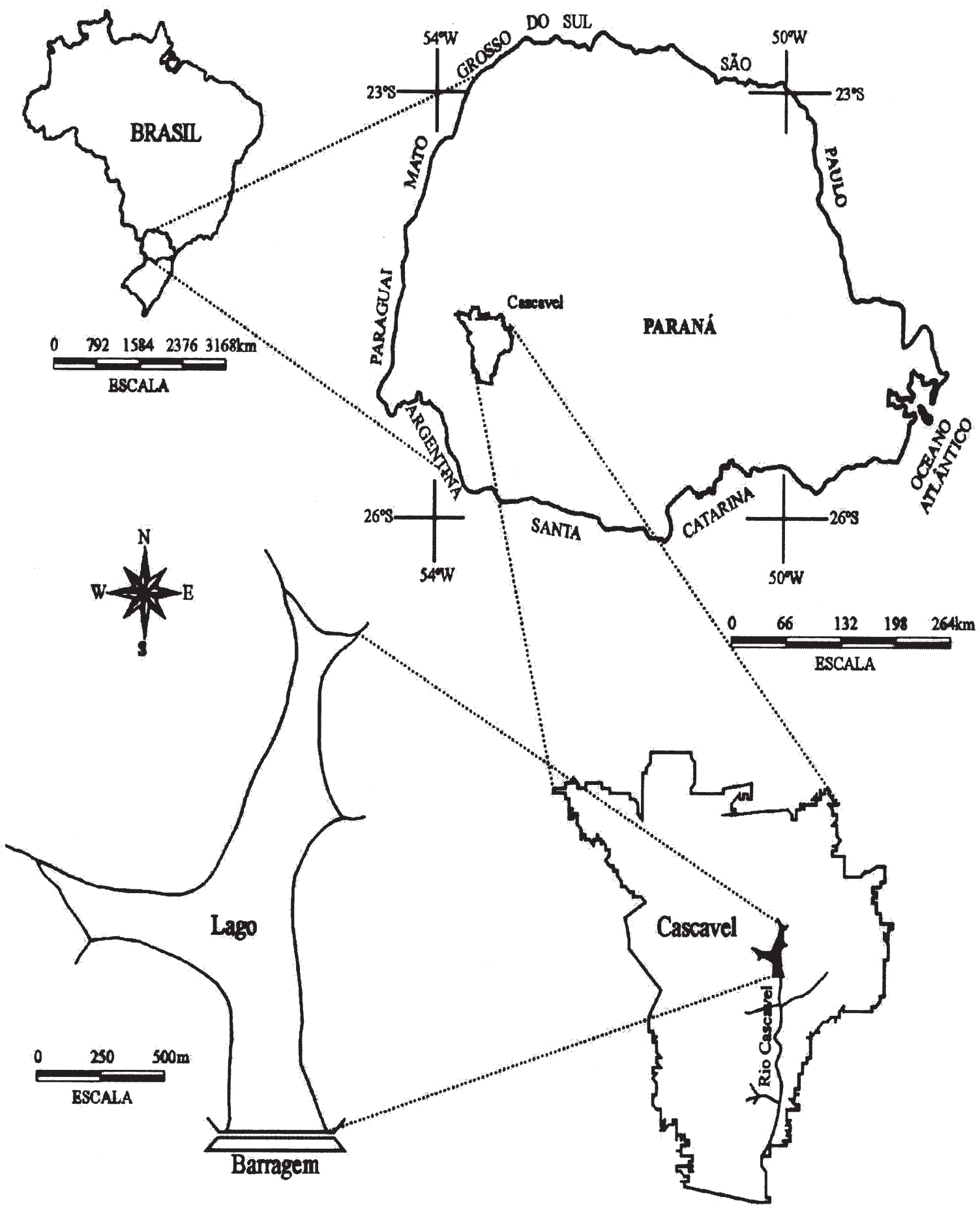

Figura 1. Localização do Lago Municipal de Cascavel, Paraná, Brasil.

Figure 1. Location of the Municipal de Cascavel Lake, Paraná, Brasil. 
Material examinado: BRASIL. PARANÁ: Cascavel, Lago Municipal de Cascavel, 26-I-2002, C. Moresco \& N.C. Bueno s.n.(HUEM11966); idem, 24-II-2002, C. Moresco \& N.C. Bueno s.n. (HUEM11967); idem, 21-VI-2002, C. Moresco \& N.C. Bueno s.n. (HUEM11971); idem, 29-IX-2002, C. Moresco \& N.C. Bueno s.n. (HUEM11974); idem, 27-X-2002, C. Moresco \& N.C. Bueno s.n. (HUEM11975); idem, 15-XII-2002, C. Moresco \& N.C. Bueno s.n. (HUEM11977); idem, 28-VI-2003, C. Moresco \& N.C. Bueno s.n. (HUEM11983); idem, 31-VIII2003, C. Moresco \& N.C.Bueno s.n. (HUEM11985); idem, 28-IX-2003, C. Moresco \& N.C. Bueno s.n. (HUEM11986).

Ocorrência no Paraná: Município de Curitiba e arredores (Lozovei \& Luz 1976); Município de Curitiba (Lozovei \& Hohmann 1977); Rio Tibagi (Bittencourt-Oliveira \& Mecenas 1994); Rio Tibagi, Município de Ponta Grossa (Bittencourt-Oliveira 2002).

Micrasterias jenneri Ralfs var. jenneri, Brit. Desm. 76. 1848.

Figura 5

Célula de contorno oblongo-elíptico, 1,4-1,5 vezes mais longa que larga, 91,8-162,1 $\mu \mathrm{m}$ compr., 67,5-107,1 um larg., istmo 17-21,6 $\mu \mathrm{m}$; semicélula 5-lobadas, incisões interlobulares lineares, estreitas, constrição mediana profunda; lobos basais e laterais cuneados a sub-retangulares; lobos basais e laterais subdivididos em 2 lóbulos retos ou chanfrados; lobo apical curto, amplamente cuneado, ápice convexo, chanfrado, ângulos sub-retangular-arredondados; parede celular lisa.

Material examinado: BRASIL. Paraná: Cascavel, Lago Municipal de Cascavel, 24-II-2002, C. Moresco \& N.C.Bueno s.n. (HUEM11967); idem, 24-V-2002, C. Moresco \& N.C. Bueno s.n. (HUEM11970); idem, 30-XI-2002, C. Moresco \& N.C. Bueno s.n. (HUEM11976).

Ocorrência no Paraná: Primeira citação da espécie.

Sormus (1991) discutiu a distinção da variedade típica da espécie e a variedade $M$.jenneri var. simplex West, sendo esta caracterizada pela ausência dos lóbulos de segunda ordem ou por apresentarem a margem externa do lóbulo levemente retusa. No entanto, conclui que este critério é instável para delimitar as duas variedades, porque pode constituir um estágio de desenvolvimento da variedade típica e é inconsistente para fins taxonômicos. Desta forma, optamos por identificar os indivíduos encontrados de acordo com Sormus (1991).

Micrasterias laticeps Nordstedt var. laticeps, Vidensk.

Meddr Naturh. Foren. 14-15: 220. 1869.

Figuras 6-7

Células de contorno elíptico, 1,2-1,3 vezes mais larga que longa, 113,5-164,8 um compr., 135,1-213,5 $\mu \mathrm{m}$ larg., istmo 17-32,4 $\mu \mathrm{m}$; semicélula 3-lobada, incisões interlobulares acutangulares, constrição mediana profunda; lobos basais semifusiformes, extremidades 2-denticuladas; lobo apical fusiforme a subcuneiforme, margem superior convexa a reta na porção mediana, ângulos acuminados, parede celular finamente pontuada.

Material examinado: BRASIL. ParanÁ: Cascavel, Lago Municipal de Cascavel, 24-II-2002, C. Moresco \& N.C.Bueno s.n. (HUEM11967); idem, 27-X-2002, C. Moresco \& N.C. Bueno s.n. (HUEM11975); idem, 15-XII-2002, C. Moresco \& N.C. Bueno s.n. (HUEM11977); idem, 27-IV-2003, C. Moresco \& N.C. Bueno s.n. (HUEM11981); idem, 27-VII-2003, C. Moresco \& N.C. Bueno s.n. (HUEM11984); idem, 28-IX-2003, C. Moresco \& N.C. Bueno s.n. (HUEM11986).

Ocorrência no Paraná: Município de Jacarezinho, Marques dos Reis (Andrade \& Rachou 1954); Município de Curitiba (Lozovei \& Hohmann 1977); Parque Regional do Iguaçu, Município de Curitiba (Picelli-Vicentim 1984); Rio Tibagi (Bittencourt-Oliveira \& Mecenas 1994); Rio Tibagi, Município de Sertanópolis (Bittencourt-Oliveira 2002); Reservatório de Rosana, Município de Diamante do Norte (Felisberto \& Rodrigues 2005); Reservatório de Salto do Vau, Município de União da Vitória (Felisberto \& Rodrigues 2005); Reservatório de Salto do Vau (Felisberto \& Rodrigues 2008); Rio São Francisco Falso, Município de Santa Helena (Biolo et al. 2008).

Trata-se de uma espécie com grande polimorfismo dos lobos basais e apicais, caracteristicamente ilustrados por Picelli-Vicentim (1984) e BittencourtOliveira \& Mecenas (1994) e verificado também no presente estudo.

Micrasterias laticeps Nordstedt var. acuminata Krieger, Rabenhorst Kryptogamen-Fl. Deutschl. 13(2): 14. 1939. 


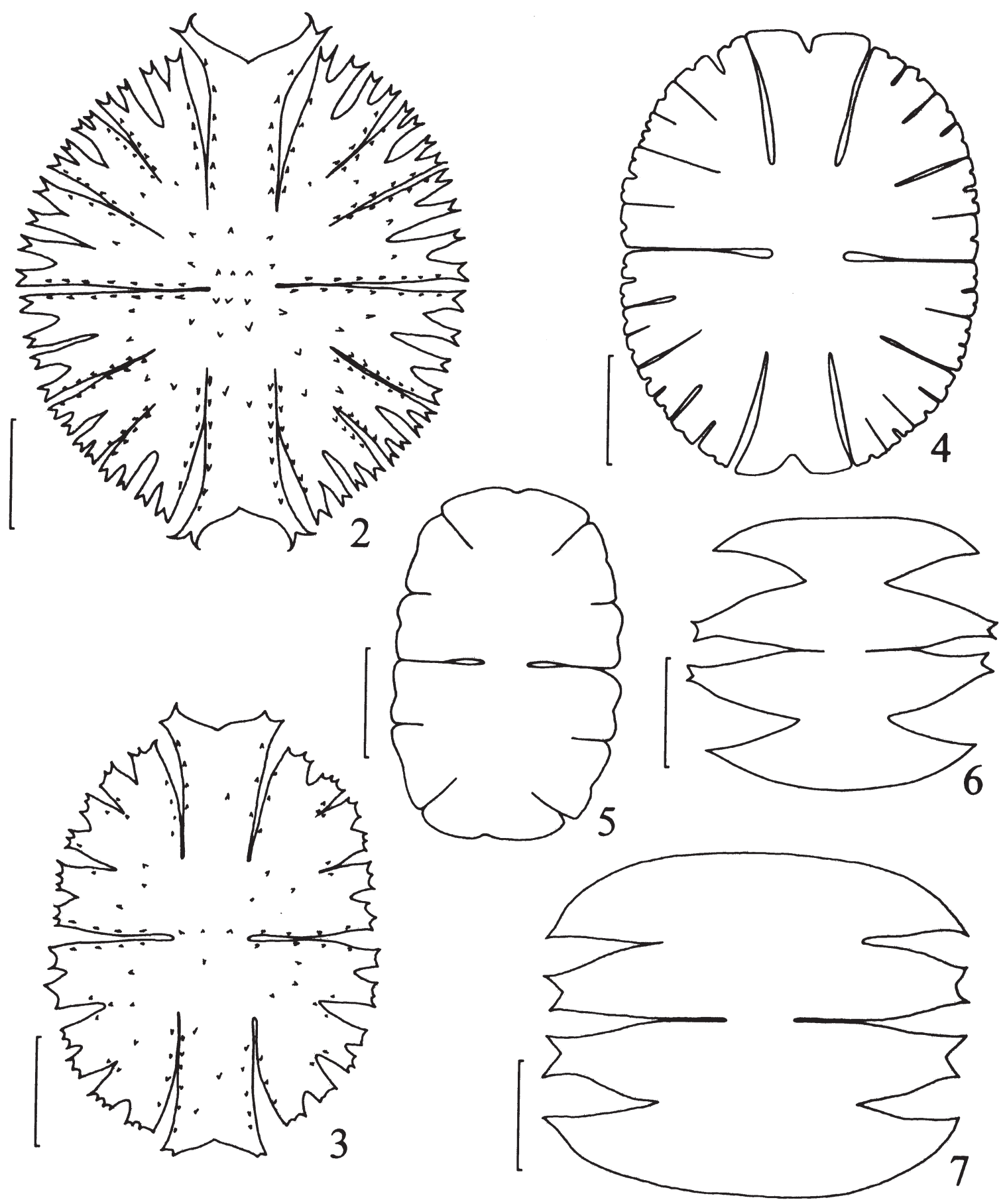

Figuras 2-7. Micrasterias no Lago Municipal de Cascavel. 2-3. Micrasterias borgei var. borgei. 4. Micrasterias denticulata var. denticulata. 5. Micrasterias jenneri var. jenneri. 6-7. Micrasterias laticeps var. laticeps. Barras de escala $=50 \mu \mathrm{m}$.

Figures 2-7. Micrasterias of the Municipal de Cascavel Lake. 2-3. Micrasterias borgei var. borgei. 4. Micrasterias denticulata var. denticulata. 5. Micrasterias jenneri var. jenneri. 6-7. Micrasterias laticeps var. laticeps. Scale bars $=50 \mu \mathrm{m}$. 
Figura 8

Célula 1,3-1,4 vezes mais larga que longa, 83,786,7 $\mu \mathrm{m}$ compr., 105,4-118,9 $\mu \mathrm{m}$ larg., istmo 20-24,3 $\mu \mathrm{m}$.

Material examinado: BRASIL. Paraná: Cascavel, Lago Municipal de Cascavel, 23-III-2002, C. Moresco \& N.C. Bueno s.n. (HUEM 11968).

Ocorrência no Paraná: Rio Cachoeira, Município de Antonina (Stankiewicz 1980); Rio Tibagi (Bittencourt-Oliveira \& Mecenas 1994); Rio Passaúna, Município de Curitiba (Cecy et al. 1997); Rio Tibagi, Município de Sertanópolis (BittencourtOliveira 2002); Reservatório de Rosana, Município de Diamante do Norte (Felisberto \& Rodrigues 2005).

Micrasterias laticeps var. acuminata difere da variedade típica da espécie por apresentar as extremidades dos lobos basais acuminadas.

Micrasterias quadridentata (Nordstedt) Grönblad, Acta Soc. Fauna Flora Fenn. 47(4): 36. 1920.

Figura 9

Célula de contorno circular ou quase, 1,1 mais longa que larga, 338-389,1 $\mu \mathrm{m}$ compr., 316,2-354 $\mu \mathrm{m}$ larg., istmo 33-67,5 $\mu \mathrm{m}$; semicélula 5-lobada, incisões interlobulares lineares, estreitas, constrição mediana profunda; lobos basais e laterais subdivididos por 3 incisões em 4 lóbulos, ápices dos lóbulos truncados, às vezes chanfrados na porção mediana, ângulos com 1 dentículo; lobo apical alongado, estreito, não estendendo-se além dos lobos laterais, margens retas ou levemente côncavas, abrindo-se para o ápice chanfrado, ângulos acuminados ou 2-denticulados; parede celular pontuada.

Material examinado: BRASIL. PARANÁ: Cascavel, Lago Municipal de Cascavel, 26-I-2002, C. Moresco \& N.C.Bueno s.n.(HUEM11966); idem, 24-II-2002, C. Moresco \& N.C. Bueno s.n. (HUEM11967); idem, 24-V-2002, C. Moresco \& N.C. Bueno s.n. (HUEM11970).

Ocorrência no Paraná: Primeira citação da espécie.

Micrasterias rotata (Greville) Ralfs var. rotata, Ann. Mag. Nat. Hist. 14: 259. 1844.

Figura 10

Célula de contorno subcircular, 1,1-1,5 vezes mais longa que larga, 202,7-324,3 um compr., 181-216,2 $\mu \mathrm{m}$ larg., istmo 27-37 $\mu \mathrm{m}$; semicélula 5-lobada, incisões interlobulares lineares, constrição mediana profunda; lobos basais e laterais cuneados, separados por uma incisão linear, profunda; lobos basais subdivididos em 4 lóbulos por 3 incisões, ápices chanfrados; lobos laterais subdivididos em 8 lóbulos por 7 incisões, ápices chanfrados ou 2-denticulados; lobo apical alongado, estreito, estendendo-se na maioria das vezes além dos lobos laterais, margens côncavas, abrindose para o ápice reto, chanfrado, margem superior emarginada a reta, ângulos levemente projetados, 2-denticulados; parede celular finamente pontuada.

Material examinado: BRASIL. PARANÁ: Cascavel, Lago Municipal de Cascavel, 26-I-2002, C. Moresco \& N.C.Bueno s.n. (HUEM11966); idem, 24-II-2002, C. Moresco \& N.C. Bueno s.n. (HUEM11967); idem, 15-XII-2002, C. Moresco \& N.C. Bueno s.n. (HUEM11977); idem, 28-IX-2003, C. Moresco \& N.C. Bueno s.n. (HUEM11986).

Ocorrência no Paraná: Município de Curitiba e arredores (Lozovei \& Luz 1976); Rio Cachoeira, Município de Antonina (Stankiewicz 1980); Rio Tibagi (Bittencourt-Oliveira \& Mecenas 1994); Rio Tibagi, Município de Sertanópolis (Bittencourt-Oliveira 2002); Reservatório de Salto do Vau, Município de União da Vitória (Felisberto \& Rodrigues 2005); Reservatório de Salto do Vau, Município de União da Vitória (Felisberto \& Rodrigues 2008); Rio São Francisco Falso, Município de Santa Helena (Biolo et al. 2008).

Micrasterias thomasiana Archer var. notata (Nordstedt) Grönblad, Acta Soc. Fauna Flora Fenn. 47(4): 38. 1920 = Micrasterias denticulata Brébisson var. notata Nordstedt, Bot. Not. 1887: 155. 1887.; K. Svenska Vet.-Akad. Handl., 4, 22(8): 29. 1888.

Figura 11

Célula de contorno subcircular, 1,1-1,5 vezes mais longa que larga, 200-324,3 $\mu \mathrm{m}$ compr., 180-216,2 $\mu \mathrm{m}$ larg., istmo 27-36 $\mu \mathrm{m}$; semicélula 5-lobada, incisões interlobulares lineares, constrição mediana profunda; lobos basais e laterais cuneados, separados por uma incisão linear, profunda; lobos basais subdivididos em 8 lóbulos por 7 incisões, ápices chanfrados; lobos laterais subdivididos em 8 lóbulos por 7 incisões, ápices chanfrados ou 2-denticulados; lobo apical alongado, estreito, estendendo-se na maioria das vezes além dos lobos laterais, margens côncavas, 

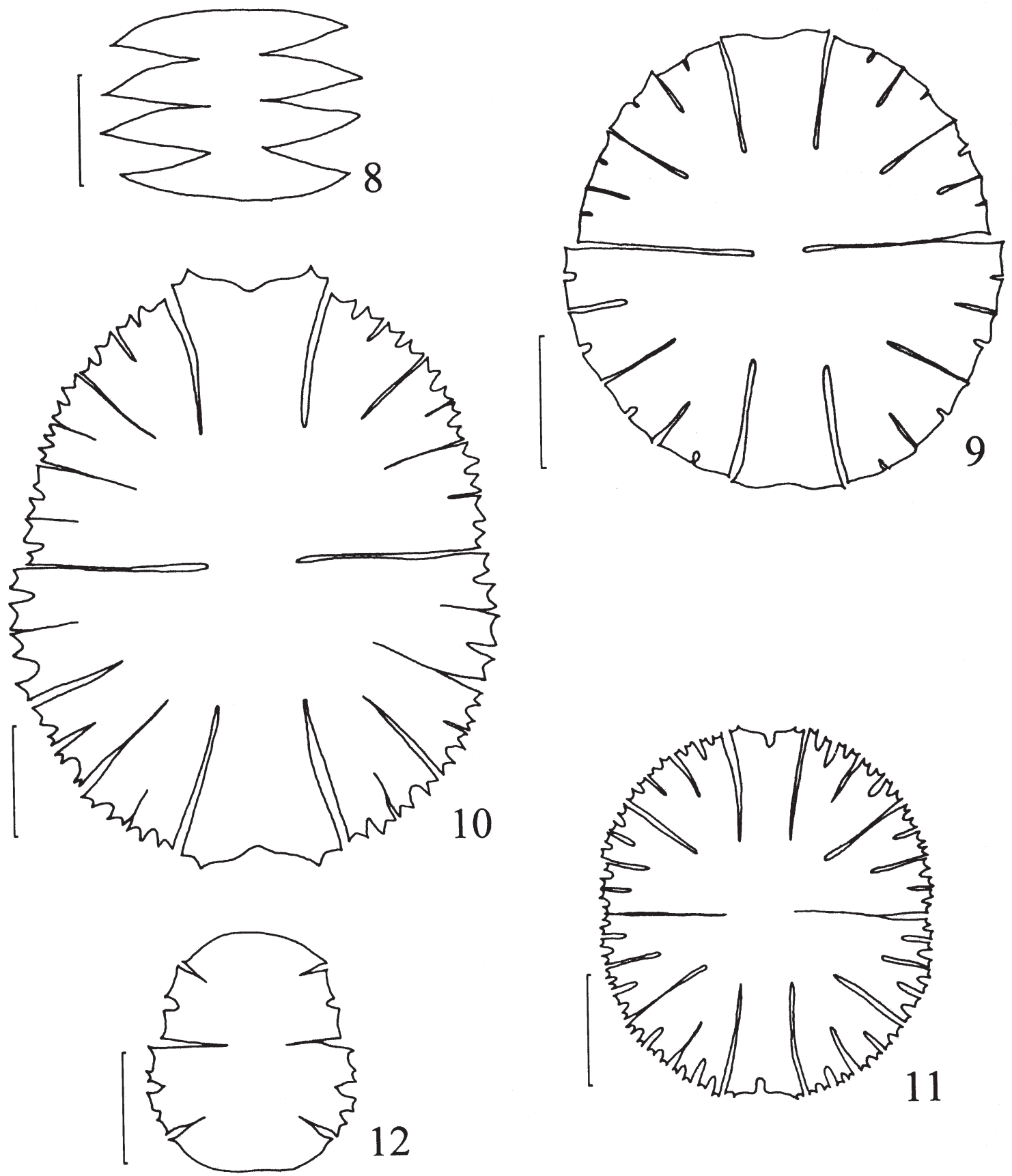

Figuras 8-12. Micrasterias no Lago Municipal de Cascavel. 8. Micrasterias laticeps var. acuminata. 9. Micrasterias quadridentata. 10. Micrasterias rotata var. rotata. 11. Micrasterias thomasiana var. notata. 12. Micrasterias truncata var. truncata . Barras de escala $=50$ $\mu \mathrm{m}(8,10,12) ; 100 \mu \mathrm{m}(9), 60 \mu \mathrm{m}(11)$.

Figures 8-12. Micrasterias in the Municipal de Cascavel Lake. 8. Micrasterias laticeps var. acuminata. 9. Micrasterias quadridentata. 10. Micrasterias rotata var. rotata. 11. Micrasterias thomasiana var. notata. 12. Micrasterias truncata var. truncata. Scale bars $=50 \mu \mathrm{m}$ $(8,10,12) ; 100 \mu \mathrm{m}(9), 60 \mu \mathrm{m}(11)$. 
abrindo-se para o ápice reto, chanfrado, incisão mediana arredondada, ângulos levemente projetados, 2-denticulados; parede celular finamente pontuada.

Material examinado: BRASIL. Paraná: Cascavel, Lago Municipal de Cascavel, 26-I-2002, C. Moresco \& N.C. Bueno s.n. (HUEM11966); idem, 21-VI2002, C. Moresco \& N.C. Bueno s.n.(HUEM11971); idem, 27-X-2002, C. Moresco \& N.C. Bueno s.n. (HUEM11975).

Ocorrência no Paraná: Parque Regional do Iguaçu, Município de Curitiba (Picelli-Vicentim 1984); Rio Tibagi (Bittencourt-Oliveira \& Mecenas 1994); Rio Tibagi, Município de Ponta Grossa, como M. thomassiana Archer var. notata (Nordstedt) Grönblad (Bittencourt-Oliveira 2002).

Difere da variedade típica da espécie por apresentar a parede celular não ornamentada com processos e espinhos e pela presença de 1-3 intumescências na porção basal da semicélula. Essas intumescências podem faltar ou serem dificilmente visíveis e não foram observadas nos táxons encontrados no presente estudo. Difere, ainda, de M.denticulata por apresentar dimensões celulares superiores.

Micrasterias truncata (Corda) Brébisson ex Ralfs var. truncata, Brit. Desm. 75. 1848.

Figura 12

Célula de contorno circular a elíptico, 1,2-1,4 vezes mais longa que larga, 100-108,1 $\mu \mathrm{m}$ compr., 70,5-93,5 um larg., istmo 23-31 um; semicélulas 5-lobada, incisões interlobulares na maioria das vezes abertas, acutangulares, constrição mediana profunda; lobos basais e laterais separados por uma incisão linear, menos profunda; lobos basais e laterais sub-retangulares, subdivididos em 2 lóbulos por 1 incisão pouco profunda, ápices chanfrados, 2-denticulados; lobo apical curto, transversalmente subfusiforme, ápice convexo ou com uma depressão leve na porção mediana, ângulos acuminados; parede celular finamente pontuada.

Material examinado: BRASIL. ParanÁ: Cascavel, Lago Municipal de Cascavel, 27-X-2002, C. Moresco \& N. C. Bueno (HUEM11975).

Ocorrência no Paraná: Rio Tibagi (BittencourtOliveira \& Mecenas 1994); Rio Tibagi, Município de Ponta Grossa e Ipiranga (Bittencourt-Oliveira 2002); Reservatório de Salto do Vau, Município de União da Vitória (Felisberto \& Rodrigues 2005).
De acordo com Bicudo \& Sormus (1982), Micrasterias truncata var. truncata consiste numa das espécies mais comuns do gênero e muitas variedades foram descritas e propostas. Bittencourt-Oliveira \& Mecenas (1994) identificaram vários morfotipos, todos circunscritos nesta variedade. Apenas um indivíduo foi encontrado durante a análise das amostras do presente estudo, contudo pôde ser identificado como M. truncata var. truncata f. truncata apresentada por Bicudo \& Sormus (1982).

\section{Literatura citada}

Algarte, V.M., Moresco, C. \& Rodrigues, L. 2006. Algas do perifíton de distintos ambientes na planície de inundação do alto rio Paraná. Acta Scientiarum (Biological Sciences) 28: 243-251.

Andrade, R.M. \& Rachou, R. 1954. Levantamento preliminar de organismos planctônicos em alguns criadouros de Anopheles darlingi, no sul do Brasil. Revista Brasileira de Malariologia 6: 481-96.

Bicudo, C.E.M. \& Menezes, M. 2006. Gêneros de algas continentais do Brasil (Chave para identificação e descrições). RiMa, São Carlos.

Bicudo, C.E.M. \& Sormus, L. 1982. Desmidioflórula Paulista, II: gênero Micrasterias C. Agardth ex Ralfs. Bibliotheca Phycologica 57: 1-230.

Biolo,S.,Siqueira, N.S.\& Bueno, N.C.2008. Desmidiaceae (exceto Cosmarium) de um tributário do Reservatório de Itaipu, Paraná, Brasil. Hoehnea 35: 145-162.

Bittencourt-Oliveira, M.C. 2002. A comunidade fitoplanctônica do rio Tibagi: uma abordagem preliminar de sua diversidade. In: M.M. Medri, E. Bianchini, O.A. Shibatta \& J.A. Pimenta (orgs.). A Bacia do rio Tibagi. FUEL, Londrina.

Bittencourt-Oliveira, M.C. \& Mecenas, P.R. 1994. Ficoflorula do Rio Tibagi, Estado do Paraná, IV: Gêneros Micrasterias, Staurastrum e Xanthidium (Zygnemaphyceae). Semina 15: 133-152.

Cecy, I.I.T., Silva, S.R.V.F. \& Boccon, R. 1997. Fitoplâncton da Represa do Rio Passaúna, Município de Araucária, Estado do Paraná. I - Divisão Chlorophyta Família Desmidiaceae. Estudos de Biologia 41: 5-32.

Felisberto, S.A. \& Rodrigues, L. 2005. Influência do gradiente longitudinal (rio-barragem) na similaridade das comunidades de desmídias perifíticas. Revista Brasileira de Botânica 28: 241-254.

Felisberto, S.A. \& Rodrigues, L. 2008. Desmidiaceae, Gonatozygaceae e Mesotaeniaceae na comunidade perifítica do reservatório de Salto do Vau (Bacia do rio Iguaçu, PR). Hoehnea 35: 71-90.

ITCF. 1990. Atlas do Estado do Paraná. Instituto de Terras, Cartografia e Florestas, Secretaria da Agricultura e Abastecimento, Curitiba. 
Lozovei, A.L. \& Hohmann, E. 1977. Principais gêneros de algas em biótopos de larvas de mosquito de Curitiba, Estado do Paraná, Brasil. III. Levantamento e Constatação da Ecologia. Acta Biológica Paranaense 6: $123-152$.

Lozovei, A.L. \& Luz, E. 1976. Diptera Culicidae em Curitiba e arredores. II Alimentação. Arquivos de Biologia e Tecnologia 19: 43-84.

Moresco, C. \& Bueno, N.C. 2007. Scenedesmaceae (Chlorophyceae - Chlorococcales) de um lago artificial urbano: Desmodesmus e Scenedesmus. Acta Scientiarum (Biological Sciences) 29: 289-296.

Picelli-Vicentim,M.M.1984.Desmídias (Zygnemaphyceae) planctônicas do Parque Regional do Iguaçu, Curitiba, estado do Paraná, Brasil: Contribuição ao levantamento.
Dissertação de Mestrado, Universidade Federal do Paraná, Curitiba.

Picelli-Vicentim, M.M., Treuersch, M. \& Leuni, L.D. 2001. Fitoplâncton da Represa do Passaúna, Estado do Paraná, Brasil. Hoehnea 28: 53-76.

Sormus, L. 1991. Desmidiaceae (Zygnemaphyceae) da Serra do Cipó, Estado de Minas Gerais, Brasil, 1: Gênero Micrasterias C. Agardh ex Ralfs. Hoehnea 18: $1-29$.

Stankiewicz, E.H. 1980. Flórula no conteúdo estomacal do Pseudocurimata gilberti (Quoy e Gaimard 1824). Tese de Doutorado, Universidade Federal do Paraná, Curitiba.

Tavares, B. \& Moreira, I.M.V. 2000. Diatomoflórula no lago artificial de Cascavel, município de Cascavel, Estado do Paraná, Brasil. Hoehnea 27: 1-24. 